\title{
A RESEARCH STUDY OF NONLINEARITY EXPERIENCING IN THE RATE OF CURRENT ACCOUNT DEFICIT TO THE BULGARIAN HEALTH AND CARE NATIONAL PRODUCT
}

\author{
Venelin Terziev ${ }^{1}$ and Stoyanka Petkova - Georgieva ${ }^{2}$ \\ ${ }^{1}$ Academician of the Russian Academy of Natural History, Moscow, Russia, \\ Prof. D.Sc. (Ec.), D.Sc. (National Security), D.Sc. (Social Activities), Ph.D., National Military \\ University, Veliko Tarnovo, Bulgaria; University of Rousse, Rousse, Bulgaria, terziev@skmat.com \\ ${ }^{2} \mathrm{PhD}$, Associate Professor, University „Prof. d-r Assen Zlatarov” - Bourgas, Bulgaria, \\ s.p.petkova@gmail.com
}

\begin{abstract}
The nonlinearity in any rate of current account deficit is an important task of research because of the socalled Regime Switching Models: TAR, SETAR, Markov Switching Model, etc. Usually, the behavior of time series exhibit breaks is associated with structural changes in government policy or financial crises. In the present research it is used as an example the calculated data about the rate of current account deficit to the Bulgarian Health and Care National Product. The series are hard to be modeling because of the structural change of the government policy about the Bulgarian Health and Care National Product. The basic hypothesis that is tested in the conducted research is that when there is a case of changes in the time series in their structure it is impossible the principles of linearity assumption to be applied. In the traditional econometrics as a science the linearity is an important assumption but there are practical evidences in which most of the time series do not provide this assumption. These cases of such time series behavior are called nonlinearity series. It is important to test the linearity assumption because of the differing between the ways of modeling the series in the case of linearity and nonlinearity using the date of the rate of current account deficit to the Bulgarian Health and Care National Product. If any series do not provide the linearity assumption and also have change in the structure then the case can be modeling with TAR, SETAR or Markov Switching Model. We provided the research by questioning whether there is nonlinearity in the rate of proportion of current account deficit to the Bulgarian Health and Care National Product and it is experienced with four nonlinearity tests: Kaplan Test, McLeod-Li test, BDS Test and Tzay Test.
\end{abstract}

Keywords: Nonlinearity Experiencing, Kaplan Test, McLeod-Li test, BDS Test and Tzay Test.

\section{INTRODUCTION}

The Health and Care organization and management is an important socio and economic activity in many countries around the world. The hospital sector in Bulgaria has traditionally been marked by overcapacity but there are real evidences for further growth. In the contemporary conditions the Bulgarian Health and Care National Product is provided by 321 hospitals with a total of just below 50000 beds. The investments for the regional hospitals depend mostly on the local municipal budgets, which usually are limited and differ according to the municipality council annual decisions for this kind of capital investments. The Bulgarian public university multi-profile hospitals for active treatment usually concentrate the capital investment in the 
inpatient sector. In 2018, the consolidated fiscal programme of the health care system has made state investigated expenditures amounting to BGN 80,9 million ( $€ 41.4$ million), out of which BGN 58,8 million ( $€$ 30,06 million) were financed by the government budget, BGN 3,7 million ( $€ 1,89$ million) from the Bulgarian National Health Insurance Fund (BNHIF), BGN 9,5 million ( $€ 4,86$ million) from the municipalities, BGN 8,5 million ( $€ 4,34$ million) from other international programmes and $B G N \quad 0,2$ million ( $€ 0,1$ million) from European sources. In comparison to 2017, the total capital was more than twice as high, amounting to BGN 185,3 million ( $€ 94,72$ million). The biggest share, BGN 133,9 million (€ 68,44 million) was financed through the EU-funded Operational Programme „Regional Development”; BGN 34 million (€17,38 million) came from the state budget and BGN 14,2 million ( $€ 7,26$ million) was provided by the Bulgarian municipalities.

Usually the structural changes in the government policy are characterized with the specific reaction on the behavior of time series exhibit break. In the present experimental research, it is used as an example the Bulgarian government policy about the Health and Care National Product according to the financial crises. It is difficult to predict the exact modeling of the series because of the structural change. When there are any changes in the structure the linearity assumption is improper to be used in this case. Linearity is an important assumption in traditional econometrics but in the practice most of the series does not provide this assumption. These series are called nonlinear series. It is important to determine the linearity assumption because it is different to modeling the series in the case of linearity and nonlinearity.

In geometry, linearity refers to Euclidean objects: lines, planes, (flat) three-dimensional space, when looked at closely enough it looks like a plane, and from a far enough distance it looks like a point. In algebra, we define linearity in terms of functions that have the property $f(x+y)=f(x)+f(y)$ and $f(a x)=a f(x)$. Nonlinearity is defined as the negative of linear. This means that the result $f$ may be out of proportion to the input $x$ or $y$. Thus, the fundamental simplifying tools of linear analysis are no longer available: for example, for a linear system, if we have two zeros, $f(x)=0$ and $f(y)=0$, then we automatically have a third zero $f(x+y)=0$. This is called the principle of superposition. Also, it means to be given many solutions from a few variables.

To explain the meaning of "non-linearity" first we must explain what the linearity means. In the literature there is lots of explanation of linearity. In the different sources the term of linearity is defined in two main subjects; linearity on variables and linearity on parameters (Walter, 2004).

In econometrics, one of the definitions of linearity is "the power of all parameters (variables) in the model must be 1 and also parameters (variables) shouldn't be the power of other parameters".

If a series does not provide the linearity assumption and also have change in the structure then it can be modeling with TAR, SETAR or Markov Switching Model. These models are called as Regime Switching Models. The regime switching model combines several sets of model parameters (coefficients) into one system, and which set of parameters should be applied depends on the regime the system is likely in at certain time.

In the present research we questioned whether there is nonlinearity in the rate of proportion of current account deficit to the Bulgarian Health and Care National Product. For this aim detecting the nonlinearity with nonlinearity test such as Kaplan Test, McLeod-Li, Tzay and BDS will be used.

\title{
2. METHODOLOGY
}

It is important in this part of the research to discuss the linearity tests. In the econometric literature there are more than 10 tests for determining the linearity. The main approach of these tests is after removing the deterministic components from the series, and then trying to find a nonlinear relation between residuals. In below some of these tests are explained. This topic has been already studied by Potter (1999) "Successful nonlinear time series modeling would improve forecasts a produce a richer notion of business cycle dynamics than linear time series models allow. For this to happen two conditions are necessary. First, economic time series must contain nonlinearities. Second, we need reliable statistical methods to summarize and understand these nonlinearities suitable for time series of the typical macroeconomic length" (Potter, 1999).

\section{THEORETICAL INTRODUCTION OF THE TEST THAT WILL BE USED FOR THE NONLINEARITY RESEARCH}

\author{
Kaplan Test
}

Kaplan test (Kaplan, 1995a) was initially formulated for the detection of determinism in the underlying dynamics of a time series, though thereafter it has been used to contrast the hypothesis of stochastic or deterministic nonlinearities in the generating process of a time series (Barnett, Gallant, Hinich, Jugeilges, Kaplan, Jensen, 1995-1997). It depends on the embedding dimension $\mathrm{m}$ and works comparing the distances 
between points in an m-dimensional reconstructed space with the distances between their images. The Kaplan's test statistic $\mathrm{K}$ is the average of the values of the distances of the images when the distances between the original points are small enough and, under the hypothesis of determinism ( ). This $\mathrm{K}$ statistic is calculated using a linear regression. This procedure involves the generation of a linear stochastic process surrogated for the original time series. With these surrogate series one can estimate the expected values for the K statistic under the hypothesis of linearity (KS). The hypothesis of linearity is rejected if the value of the test statistic from the surrogates is never small enough relative to the value of the statistic computed from the original data $(\mathrm{K}<\mathrm{KS})$.

\section{McLeod-Li Test}

As it is understood from it is name the test was proposed by McLeod and Li in 1983. In this test the authors proposed that if there is a statistically significant autocorrelation in square of residuals which obtained from a linear equation.

McLeod-Li test is used to check if there is presence of nonlinearity in variance. The test uses the squared residual of the prewhitened time series, showing that under the null hypothesis of linearity (concretely variance linearity), the autocorrelation function should be zero in all time lags. Under that null hypothesis, for a lag big enough, the statistic $Q=N(N+2) \sum_{i=1}^{L} \hat{r}_{k}^{2} /(N-i)$ follows a $\chi^{2}(L)$ where $\hat{r}_{k}^{2}$ is the estimated autocorrelation function for the squared residual.

The linear serial dependence is removed from the data through a pre-whitening model in the following way: Firstly, we fit an $\operatorname{AR}(p)$ model to the sample data for values from $p=0$ to $p=12$. The optimal lag is chosen to minimize the Schwartz criterion (SC) and Akaice Info Criteria (AIC). The Schwartz criterion is known to be consistent for $\operatorname{AR}(p)$ order determination under the null hypothesis of a linear generating mechanism compared to alternative choices (Brock, Hsieh, LeBaron, 2011; Cromwell, Labys, Teraza, 2014). The residuals of the $A R(p)$, $\{e t\}$, which are by construction serially uncorrelated, are then tested for non-linear independence using each of the procedures. Other specifications such as ARMA or GARCH could be used as an alternative pre-whitening model.

This test for ARCH effects was proposed by McLeod and Li (1983) (McLeod, 2013a) based on a suggestion in Granger and Andersen (1978) (Hinich, Patterson, 2015). It looks at the autocorrelation function of the squares of the prewhitened data and tests whether $\operatorname{corr}\left(x_{t}^{2}, x_{t-k}^{2}\right)$ it is non-zero for some $\mathrm{k}$. The autocorrelation function for the squared residuals $\left\{x_{t}^{2}\right\}$ is estimated by:

(1) $\hat{r}(k)=\frac{\sum_{t=k+1}^{T}\left(x_{t}^{2}-\hat{\sigma}^{2}\right)\left(x_{t-k}^{2}-\hat{\sigma}^{2}\right)}{\sum_{t=1}^{T}\left(x_{t}^{2}-\hat{\sigma}^{2}\right)}$, where

(2) $\hat{\sigma}^{2}=\sum_{t=1}^{T} \frac{x_{t}^{2}}{T}$

Under the null hypothesis that $\mathrm{x}$ is an independent and identically distributed (i.i.d) process McLeod and Li show that for fixed $L$

(3) $\sqrt{T} \hat{r}=(\hat{r}(1), \ldots . \hat{r}(L))$ is asymptotically a multivariate unit normal. Consequently, the usual Box-Ljung statistic

(4) $Q=T(T+2) \sum_{i=1}^{L} \frac{\hat{r}^{2}(k)}{T-i}$ is asymptotically $\chi^{2}(L)$ under the null hypothesis of a linear generating mechanism for the data (Ashley, Patterson, 1998).

Engle LM Test

The Engle test which detects ARCH disturbances is based on the same idea with McLeod-Li test but it uses the determination coefficient $R^{2}$ of an auxiliary regression of the equation: $e_{t}^{2}=\alpha_{0}+\sum_{i=1}^{p} \alpha_{k} e_{t-1}^{2}+\varepsilon_{t}$. Bollerslev (1986) suggests that it should also have power against GARCH alternatives (Granger, Terasvirta, 2013; Hinich, Patterson, 2015). Since it is a Lagrange Multiplier test, the test statistic itself is based on $R^{2}$ of an auxiliary regression, which in this case can be defined as: 
(5) $e_{t}^{2}=\alpha_{0}+\sum_{i=1}^{p} \alpha_{k} e_{t-i}^{2}+v_{t}$

Under the null hypothesis of a linear generating mechanism for $e_{t}, N R^{2}$ for this regression is asymptotically $\chi^{2}(p)$.

\section{BDS Test}

The BDS test was suggested by Brock, Dechert, \& Scheinkman 1987 (Barnett, Gallant, Hinich, Jugeilges, Kaplan, Jensen, 1995). It is a test of independence, but it can be used to produce indirect evidence of nonlinearity if all linear dependence has already been removed. Under the null hypothesis of i.i.d., the authors derive a statistic which depends on the embedding dimension $m$ that follows asymptotically the standard normal distribution.

The BDS test is employed to throw some additional light on to the features of data generating process. The applied way is the same. If the linear structures removed from the series by ARMA models so that the residuals cannot be distinguished from white noise realizations by traditional time series diagnostics. There will be still any nonlinear structures left which will prove to be statistically significant.

The test uses the correlation function which is based on all possible comparisons of $\mathrm{m}$-dimensional vectors (lags of series), such as $X_{t}^{m}=\left(x_{t}, x_{t+1} \ldots . . x_{t+m-1}\right)$, or of observations of a considered time series.

The BDS statistic tests the null hypothesis that the elements of a time series are independently and identically distributed (IID). For a time, series which is IID, the distribution of the statistic:

(6) $W_{m}(\varepsilon)=\frac{\sqrt{n}\left(C_{m}(\varepsilon)-C_{1}(\varepsilon)^{m}\right)}{\sigma_{m}(\varepsilon)}$

$W_{m}(\varepsilon)$ is known as the BDS statistic. $C_{m}(\varepsilon)$ denotes the fraction of $m$-tuples in the series, which are within a distance of each other and $\sigma_{m}(\varepsilon)$ is an estimate of the standard deviation under the null hypothesis of IID. The test statistic is asymptotically standard normal under the null of whiteness. The null is rejected if the test statistic is absolutely large, (say greater than 1.96). If the null hypothesis of IID cannot be accepted this implies that the residuals contain some kind of hidden structure, which might be non-linear - or even chaotic.

BDS has its origin in the empirical literature on testing for low-dimensional chaos in economic and financial data. Suggested among other tests for non-linearity by Lee et al. (1993) this test seems to have reasonable power, but only in samples of at least 200 observations. That is not our case, nor the case of most macroeconomic series, unless monthly data are available for a long time. Nevertheless, this test is used in Altug et al. (1999) and in Stanca (1999), which have only quarterly data.

\section{Tzay Test}

This test was suggested by Tzay (1986) (Tzay, 2016). It is a generalization of the Keenan (1985) test. It explicitly looks for quadratic serial dependence in the data. Let $K=k(k-1) / 2$ column vectors $V_{1}, \ldots V_{k}$ contain all of the possible cross-products of the form $e_{t-i} e_{t-j}$, where $i \in[1, k]$ and $j \in[i, k]$. Thus, $v_{t, 1}=e_{t-1}^{2}, v_{t, 2}=e_{t-1} e_{t-2}$, $v_{t-3}=e_{t-1} e_{t-3}, \quad v_{t, k+1}=e_{t-2} e_{t-3}, \quad v_{t, k+2}=e_{t-2} e_{t-4}, \ldots \ldots, \quad v_{t, k}=e_{t-k}^{2}$. And let $\hat{v}_{t-j}$ denote the projection of $v_{t, i}$ on the orthogonal subspace $e_{t-1}, \ldots ., e_{t-k}$, (i.e. the residuals from a regression of $v_{t j}$ on $e_{t-1}, \ldots \ldots e_{t-k}$. The parameters $\gamma_{1}, \ldots . . \gamma_{k}$ are then estimated by applying OLS to the regression equation

(7) $e_{t}=\gamma_{0}+\sum_{i=1}^{K} \gamma_{i} \hat{v}_{t, i}+\eta_{t}$

Note that the $j$ th regressor in this equation is $\hat{v}_{t, j}$, the period $t$ fitting error from a regression of $v_{i, t}$ on $e_{t-1}, \ldots, e_{t-k}$. So long as $p$ exceeds $K$, this projection is unnecessary for the dependent variable $\{e t\}$ if it is prewhitened using an $\operatorname{AR}(p)$ model. The Tzay test statistic then is just the usual $F$ statistic for testing the null hypothesis that $\gamma_{1}, \ldots . \gamma_{k}$ is all zero. 


\section{CALCULATING THE DATA, EXAMINING THE MODELS, NONLINEARITY AND OBTAINING THE RESULTS OF THE PRESENT RESEARCH}

In the present research we analyzed the rate of current account deficit to the Bulgarian Health and Care National Product. The obtained results are from a monthly data between 2004.12- 2018.12. In Figure 1 are graphically represented the results from the tests series. The date was calculated by the mathematical programs E - views 6.4, Rates 6.0 and Math Lab by which was detect the type of series.

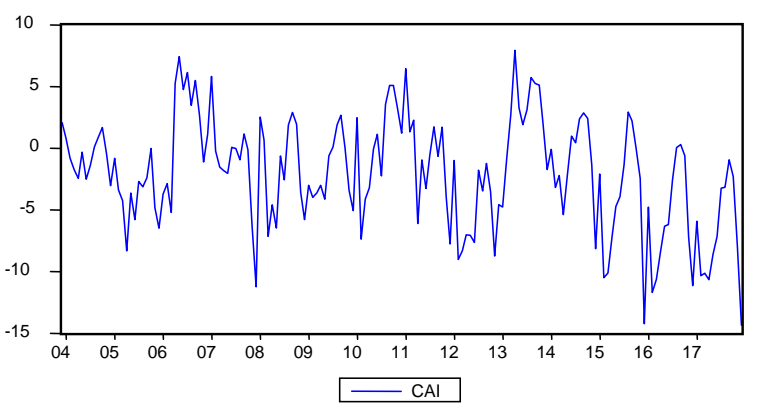

Fig. 1: The results from the tests series

After purifying the deterministic events from the series, the view of the series is given in Figure 2.

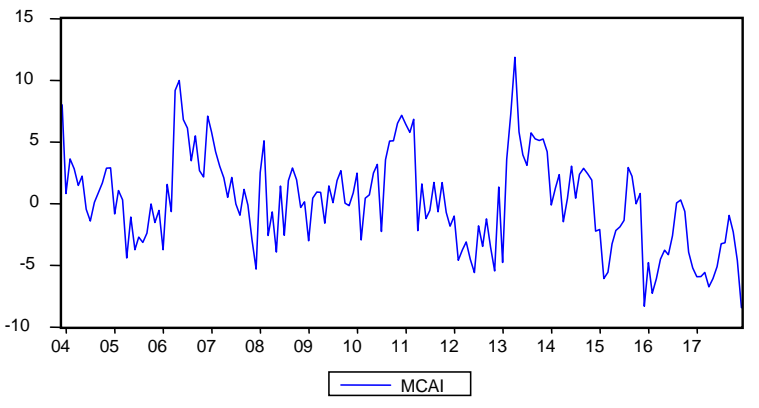

Fig. 2: The view of the series

The examined series were used to determine if there is linear or nonlinear co-relation as it is shown on Figure 2.

With no constant we calculated and obtained the results as shown on Table 1:

Table 1: The results from examined series

\begin{tabular}{|c|c|c|c|c|c|c|c|c|c|c|c|c|}
\hline MA & AR & 1 & 2 & 3 & 4 & 5 & 6 & 7 & 8 & 9 & 10 & 11 \\
\hline 1 & 4.8173 & 4.7978 & 4.7973 & 4.8248 & 4.8412 & 4.8599 & 4.8703 & 4.8845 & 4.9003 & 4.9191 & 4.9356 & 4.9437 \\
\hline 2 & 4.8132 & 4.8087 & 4.8158 & 4.8343 & 4.8524 & 4.8702 & 4.8299 & 4.8463 & 4.8471 & 4.8788 & 4.9200 & 4.9431 \\
\hline 3 & 4.8240 & 4.8205 & 4.8279 & 4.7848 & 4.7885 & 4.7844 & 4.8605 & 4.8679 & 4.8774 & 4.8401 & 4.8557 & 4.9447 \\
\hline 4 & 4.8359 & 4.8301 & 4.8339 & 4.7981 & 4.8431 & 4.8238 & 4.7586 & 4.8909 & 4.9013 & 4.7838 & 4.8702 & 4.9109 \\
\hline 5 & 4.8359 & 4.8301 & 4.8399 & 4.7981 & 4.8543 & 4.8287 & 4.8526 & 4.8385 & 4.7800 & 4.8554 & 4.9130 & 4.9062 \\
\hline 6 & 4.8532 & 4.8636 & 4.8118 & 4.8138 & 4.8746 & 4.8376 & 4.7535 & 4.7791 & 4.9370 & 4.8635 & 4.9158 & 4.9061 \\
\hline 7 & 4.8632 & 4.8544 & 4.8568 & 4.8589 & 4.7915 & 4.8527 & 4.7685 & 4.7947 & 4.8366 & 4.8670 & 4.9116 & 4.9628 \\
\hline 8 & 4.8558 & 4.8551 & 4.8719 & 4.8595 & 4.8125 & 4.9231 & 4.7871 & 4.9040 & 4.8947 & 4.8773 & 4.8938 & 4.8675 \\
\hline 9 & 4.8662 & 4.8657 & 4.8348 & 4.8395 & 4.8497 & 4.8574 & 4.7951 & 4.9154 & 4.8952 & 4.8800 & 4.9108 & 4.8310 \\
\hline
\end{tabular}


IJASOS- International E-Journal of Advances in Social Sciences, Vol. V, Issue 14, August 2019

\begin{tabular}{|c|c|c|c|c|c|c|c|c|c|c|c|c|}
\hline 10 & 4.8781 & 4.8756 & 4.8470 & 4.8485 & 4.8606 & 4.8976 & 4.9049 & 4.8309 & 4.9049 & 4.8167 & 4.9225 & 4.6223 \\
\hline 11 & 4.8825 & 4.8809 & 4.8568 & 4.8582 & 4.8810 & 4.8959 & 4.8179 & 4.8523 & 4.9147 & 4.8725 & 4.9344 & 4.8872 \\
\hline 12 & 4.8973 & 4.8835 & 4.8641 & 4.8252 & 4.8502 & 4.9048 & 4.9099 & 4.8429 & 4.9041 & 4.8118 & 4.9108 & \\
\hline
\end{tabular}

With constant we calculated and obtained the results as shown on Table 2:

Table 2: The results from examined series

\begin{tabular}{|l|l|l|l|l|l|l|l|l|l|l|l|l|}
\hline MA & AR & 1 & 2 & 3 & 4 & 5 & 6 & 7 & 8 & 9 & 10 & 11 \\
\hline 1 & 4.8289 & 4.8097 & 4.8262 & 4.8368 & 4.8533 & 4.8720 & 4.8824 & 4.8968 & 4.9157 & 4.9315 & 4.9479 & 4.9558 \\
\hline 2 & 4.8249 & 4.8206 & 4.8278 & 4.8463 & 4.8644 & 4.8824 & 4.7511 & 4.7499 & 4.8944 & 4.8802 & 4.9324 & 4.9553 \\
\hline 3 & 4.8357 & 4.8324 & 4.8398 & 4.7975 & 4.8024 & 4.7252 & 4.8725 & 4.8800 & 4.8988 & 4.8541 & 4.7399 & 4.9575 \\
\hline 4 & 4.8475 & 4.8419 & 4.8518 & 4.8078 & 4.8552 & 4.8382 & 4.7610 & 4.8919 & 4.8932 & 4.7083 & 4.8821 & 4.9285 \\
\hline 5 & 4.8534 & 4.8536 & 4.8638 & 4.8222 & 4.8582 & 4.8487 & 4.8650 & 4.7572 & 4.7572 & 4.8686 & 4.9267 & 4.9184 \\
\hline 6 & 4.8647 & 4.8553 & 4.8240 & 4.8252 & 4.8585 & 4.8480 & 4.6948 & 4.8888 & 4.9186 & 4.8760 & 4.9277 & 4.9297 \\
\hline 7 & 4.8750 & 4.8661 & 4.8686 & 4.8709 & 4.8786 & 4.7887 & 4.7185 & 4.9018 & 4.7996 & 4.8917 & 4.9682 & 4.8249 \\
\hline 8 & 4.8675 & 4.8669 & 4.8838 & 4.8711 & 4.8274 & 4.7049 & 4.7339 & 4.9165 & 4.9086 & 4.8591 & 4.9078 & 4.8581 \\
\hline 9 & 4.8779 & 4.8776 & 4.8469 & 4.8505 & 4.8625 & 4.8659 & 4.7505 & 4.9279 & 4.9084 & 4.9131 & 4.9235 & 4.8136 \\
\hline 10 & 4.8898 & 4.8875 & 4.8587 & 4.8638 & 4.8472 & 4.8977 & 4.7522 & 4.7994 & 4.9151 & 4.7957 & 4.9396 & 4.8948 \\
\hline 11 & 4.8969 & 4.8928 & 4.8703 & 4.8699 & 4.8939 & 4.9074 & 4.7690 & 4.8659 & 4.7734 & 4.8863 & 4.9443 & 4.8676 \\
\hline 12 & 4.9086 & 4.8950 & 4.8764 & 4.8620 & 4.8591 & 4.9150 & 4.9181 & 4.8569 & 4.9152 & 4.8098 & 4.9255 & \\
\hline
\end{tabular}

Examining the models and the presence of nonlinearity by Kaplan Test

We make the suggestions that the null hypothesis indicates whether the series are linear and the alternative hypothesis indicates that the series are nonlinear and these are defined as fallowed:

- $\mathrm{H}_{0}$ : Time series are Linear;

- $\mathrm{H}_{1}$ : Time series are Non linear.

The criterion to reject the null hypothesis is that the test statistic value will never smaller than minimum value of the surrogates' data. Reject the null hypothesis for all level of embedding dimension. Table 3 shows minimum, mean, standard deviation, embedding dimension, test statistic, and conclusion of the Kaplan test statistic. As shown in Table 3 the linearity assumption is rejected.

Table 3: The Kaplan test statistic

\begin{tabular}{|c|c|c|c|c|c|c|}
\hline $\begin{array}{c}\text { Embedding } \\
\text { Dimension }\end{array}$ & Mean & Minimum & Max & Standard Deviation & Test Statistic & Conclusion \\
\hline 2 & 3.93 & 2.76 & 4.89 & 0.3695 & 3.46 & Reject Linearity \\
\hline 3 & 4.06 & 2.66 & 5.79 & 0.6076 & 3.43 & Reject Linearity \\
\hline 4 & 3.79 & 1.72 & 5.97 & 0.7812 & 3.86 & Reject Linearity \\
\hline 5 & 3.73 & 1.87 & 5.77 & 0.7652 & 2.98 & Reject Linearity \\
\hline
\end{tabular}


Examining the models and the presence of nonlinearity by McLeod-Li Test

In McLeod-Li test it is searched that if there is evident autocorrelation between residual from obtained a linear equation. For the application of McLeod-Li test, we used the AR (1) model. After the estimation of AR (1) model, the residuals are obtained and the new model is established as:

(8) $\hat{e}_{t}^{2}=\alpha_{0}+\alpha_{1} \hat{e}_{t-1}^{2}+\ldots . \alpha_{n} \hat{e}_{t-n}^{2}+v_{t}$

If there is no linearity, all the coefficients are becoming equal to zero, that is: $\alpha_{1}=\ldots \ldots . \alpha_{n}=0$. The model is:

(9) $\hat{e}_{t}^{2}=5.584003983+0.194886039 * \hat{\mathrm{e}}_{\mathrm{t}-1}^{2}$

And the hypothesis can be tested using standard F-test and for $\mathrm{AR}(1)$ model, the calculated F-statistics are given below: F-statistic: 6,355307; Prob (F-statistic): 0,012658

In this case the linearity assumption is rejected and it can be said that there is nonlinearity in the series.

The coefficient obtained from the test is differing from 0 so that it can be seen that there is nonlinearity.

Examining the models and the presence of nonlinearity by the BDS Test.

In BDS test the null hypothesis says that the examined series is iid so that the hypotheses are constructed as fallowed:

- $\mathrm{H}_{0}$ : Error terms are iid;

- $\mathrm{H}_{1}$ : Error terms are not iid.

Table 4 shows the test statistics values of BDS test. Critical value for $\alpha=0.05$ is equal to 2.33. At the third embedding dimension all level of $\varepsilon$ null hypothesis is rejected. That means errors are dependent either there exist nonlinearity or chaotic dependence.

Table 4: The statistics values of BDS test

\begin{tabular}{|c|c|c|c|c|c|c|c|}
\hline & \multicolumn{7}{|c|}{ Embedding Dimension } \\
\hline$\varepsilon$ & 2 & 3 & 4 & 5 & 6 & 7 & 8 \\
\hline 0.5 & 0.87 & 2.41 & 2.77 & 0.88 & 1.06 & 0.99 & 1.42 \\
\hline 0.75 & 1.46 & 2.68 & 1.97 & 0.58 & -0.56 & -1.27 & -1.40 \\
\hline 1 & 1.23 & 2.44 & 1.88 & 1.39 & 0.91 & 0.40 & 0.00 \\
\hline 1.5 & 1.66 & 2.33 & 2.21 & 1.94 & 1.95 & 1.08 & 0.86 \\
\hline 1.75 & 1.65 & 2.36 & 2.12 & 1.93 & 1.61 & 1.27 & 1.10 \\
\hline 2 & 2.10 & 2.62 & 2.33 & 2.12 & 1.77 & 1.40 & 1.25 \\
\hline
\end{tabular}

As a result, that we conclude that null hypothesis is not rejected for all level of embedding dimension and epsilon level.

1. Test statistics is calculated from the time series which is shown in point 3.

2. Create 100 obtained data, which is random linear time series has the same mean, variance and autocorrelation function with the time series, test statistics are calculated for these surrogates.

3. Linearity is rejected, if the value of the test statistic from the time series is not smaller than the test statistic computed from the obtained data.

Examining the models and the presence of nonlinearity by the Tzay Test

In the Tzay test in all cases except the 3 threshold and the delay for 3 and 4 , all test statistics shows that the series is linear. 
Table 5: The results from Tzay test

\begin{tabular}{|c|c|c|c|}
\hline & Delay & $F$ Statistics & Probability \\
\hline Threshold Variable 1 & 1,2 & $F(3,161)=2.49$ & $P=0.06$ \\
\hline & $1,2,3$ & $F(3,160)=0.52$ & $P=0.66$ \\
\hline Threshold Variable 2 & $1,2,3,4$ & $F(3,159)=0.34$ & $P=0.79$ \\
\hline & 2,3 & $F(3,160)=1.82$ & $P=0.84$ \\
\hline & $2,3,4,5$ & $F(3,158)=0.38$ & $P=0.76$ \\
\hline Threshold Variable 3 & $3,3,4,5,6$ & $F(3,157)=0.29$ & $P=0.83$ \\
\hline & $3,4,5$ & $F(3,158)=0.57$ & $P=0.02$ \\
\hline & $3,4,5,6$ & $F(3,157)=0.53$ & $P=0.65$ \\
\hline Threshold Variable 4 & 4,5 & $F(3,158)=1.16$ & $P=0.32$ \\
\hline & $4,5,6$ & $F(3,157)=0.43$ & $P=0.72$ \\
\hline
\end{tabular}

\section{CONCLUSION}

The Bulgarian Health and Care product as a complex socio-economic „phenomenon” has multilateral significance. Generally, this can be characterized by its meaning of a "healthy importance" - it grows with the development of industrialization and urbanization, with the stress and immobilization characteristic of the lifestyle and labor activity of the contemporary society. This is as a result of the active lifestyle of living that the "modern man" has the opportunity to improve the functional health state of his organism (Sotirov, Sakakushev, Koriykov, Georgiev, Zhel, Popesku, 2010a).

To conclude, we can say that rate of current account deficit to the Bulgarian Health and Care National Product between 2004.12 and 2018.12 can be characterized by the followed conclusions:

- It has a nonlinear deterministic behavior with some level of noise;

- Testing the series with the McLeod- Li test resulted the series is nonlinearity;

- Testing it with the BDS test only the third embedding dimension is nonlinear with all types of .

- Testing it with the Kaplan test the obtained series is nonlinear

- Testing the series with the Tzay test it can seem that the series is linear except 3 thresholds and for the delay 3 and 4 .

Although the tests examine the different types of nonlinearities the result is neither the series is linear or nonlinear. The type of nonlinearity is not the same at all series.

As a result, we conclude that, because of the different nonlinearity structure of the series, it must be used more than one nonlinearity test to arrive the true conclusion. Besides it is necessary to modeling that the series is linear or nonlinear. If the series is linear, it is suitable to use linear models such as AR, MA, ARMA models and if the series is nonlinear, it is suitable to use nonlinear models such as Threshold AR, Markov Switching or Self Exciting TAR models.

\section{REFERENCE LIST}

Ashley, R.A., Patterson, D.M., (1998). Nonlinear Model Specifications/Diagnostics: Insights from a Battery of 
Nonlinearity Tests, 1998.

Barnett, W.A., Gallant, R.A., Hinich, M.J., Jugeilges, A.J., Kaplan, D.T., Jensen, M.J., (1995), Robustness of Nonlinearity and Chaos Test to Measurement Error, Inference Method and Sample Size, Journal of Economic Behavior and Organization, 27, 301-320, 1995.

Barnett, W.A., Gallant, R.A., Hinich, M.J., Jugeilges, A.J., Kaplan, D.T., Jensen M.J., (1996). An Experimental Desing To Compare Tests of Nonlinearity and Chaos, In Barnett W.A Kirman,A.P. and Salmon M. (Editors) Journal of Econometrics 77, 297-302, 1996.

Barnett, W.A., Gallant, R.A., Hinich, M.J., Jugeilges, A.J., Kaplan, D.T., Jensen M.J., (1997). A Single Blind Controlled Competition among Test for Nonlinearity and Chaos, Journal of Econometrics, 77, 297-302, 1997.

Brock, W.A, Hsieh, D.A., LeBaron, B, (2011). Nonlinear Dynamics, Chaos and Instability: Statistical Theory and Economic Evidence, MIT Press, Cambridge, Massachusetts, 2011.

Cromwell, JB, Labys, WC., Teraza, M (2014), Univariate Tests for Time Series Models Sage, Thousand Oaks, Canada p.44, 2014.

Granger, C.W.J., Terasvirta, T. (2013), Modeling Nonlinear Economic Relationships, Oxford University Press, 2013.

Granger, C.W.J. (2010). Overview of Nonlinear Time Series Specifications in Economics, 2010.

Hinich, M., Patterson, D.M. (2015), Detecting Epochs of Transient Dependence in White Noise, University of Texas at Austin, 2015.

Kaplan, D.T. (1995a), Nonlinearity and No stationary: The Use of Surrogate Data in Interpreting Fluctuations, Center of Nonlinear Dynamics and Dept of Physiology McGill University Montreal, Canada, 1995.

McLeod, Li (2013a), Diagnostic Checking of ARMA time Series Models Using Squared- Residual Autocorrelation, Journal of Time Series Analysis 4, 269-273, 2013.

Patterson, D.M. and Ashley, R. (2000). A Nonlinear Time Series Workshop, Kluwer, Norwell, 2000.

Sotirov, B., Sakakushev, B., Koriykov, Ts., Georgiev, Ts, Zhel, Zh. F, Popesku, G. (2010a). „Menidzhmant na kachestvoto i metrologiya" - spetsialnost ot evropeyskoto obrazovatelno prostranstvo. // Standartizatsiya, Metrologiya, Sertifikatsiya, 2010, broy I, str. 25-28, ISSN 1310-0831 (Сотиров, Б., Сакакушев, Б., Корийков, Ц., Георгиев, Ц, Жел, Ж. Ф, Попеску, Г. „Мениджмънт на качеството и метрология“ - специалност от европейското образователно пространство. // Стандартизация, Метрология, Сертификация, 2010, брой I, стр. 25-28, ISSN 1310-0831).

Potter, S.M., (1999). Nonlinear Impulse Response Functions, Federal Reserve Bank of New York. http://www.newyorkfed.org/research/staff_reports/sr87.pdf, 1999.

Tzay, R. S, (2016). Nonlinearity Tests for Time Series, Biometrika 73, 461-466, 2016.

Walter, E., Applied Econometrics Time Series, (2004). Second Edition p. 406, 2004. 